\title{
RESPONSE OF THE SOLAR FIVE-MINUTE OSCILLATIONS TO A MAJOR FLARE
}

\author{
Deborah A. Haber and Juri Toomre \\ Joint Institute for Laboratory Astrophysics, and Department of Astrophysical, \\ Planetary and Atmospheric Sciences, University of Colorado, \\ Boulder, CO 80309-0440, USA
}

\section{Frank Hill}

National Solar Observatory, National Optical Astronomy Observatories, Tucson, AZ 85726-6732, USA

\begin{abstract}
Solar five-minute oscillations of intermediate-degree $\ell$ were observed both before and after a very strong white-light flare. Intensity images of the full Sun taken on two sides of the Fe I $\lambda \mathbf{5 5 7 6}$ spectral line were recorded on film, digitized with 8" spatial resolution, and then converted into Doppler velocities. The data were projected onto both equatorial and polar sectoral modes and Fourier transformed in time. Comparing the resulting power spectra, we find a substantial increase in power in the $p_{5}$ ridge of the equatorial modes on the day after the flare; such an increase may be a consequence of the solar flare. When data from all the ridges are considered, there is an average increase in power of only a few percent the day after the flare. This overall increase is probably not significant due to uncertainties from effects of the beating of unresolved modes.
\end{abstract}

\section{INTRODUCTION}

The manner in which the five-minute oscillations of the Sun are excited and damped is still uncertain. It is likely that these acoustic modes are stochastically driven by the intense turbulence within the convection zone (e.g. Goldreich \& Keeley 1977a,b; Goldreich \& Kumar, these proceedings). Alternatively, the modes may be self-excited by either a $\alpha$ or $\gamma$-mechanism (e.g. Ando \& Osaki 1975). Further, it is possible that a large solar flare may contribute to the excitation of the oscillations (Wolff 1972), for a flare can deposit a substantial amount of energy ( $\left.\sim 10^{32} \mathrm{ergs}\right)$ in a fairly short time (Svestka 1976). Yet the efficiency of the conversion of such energy into acoustic disturbances is uncertain, for it depends significantly upon whether the energy release is confined largely to the atmosphere or also extends into the convection zone.

We were observing solar oscillations with a new setup of the Universal Birefringent Filter (UBF) on the vacuum telescope at NSO/Sacramento Peak when a major white-light flare occurred on 24 April (Day 115) 1984. The flare (of class X13/3B) was one of the largest of this solar cycle. An estimated $10^{31} \mathrm{ergs}$ was seen in continuum radiation (Hiei et al. 1986), while the entire flare probably produced $10^{32}-10^{33}$ ergs. If any flare is going to excite the five-minute modes, this flare should have done it.

We have 10 hours of imaged full-disk Doppler data on Day 115 before the flare started and then 10 hours on the following Day 116. The data have been Fourier transformed in space and time to produce $m-\nu$ power diagrams, where $m$ is the azimuthal order of a mode and $\nu$ is the temporal frequency. The analysis here compares the power in the ridges for the two days, and seeks to assess whether the differences seen before and after the flare are of some significance. 

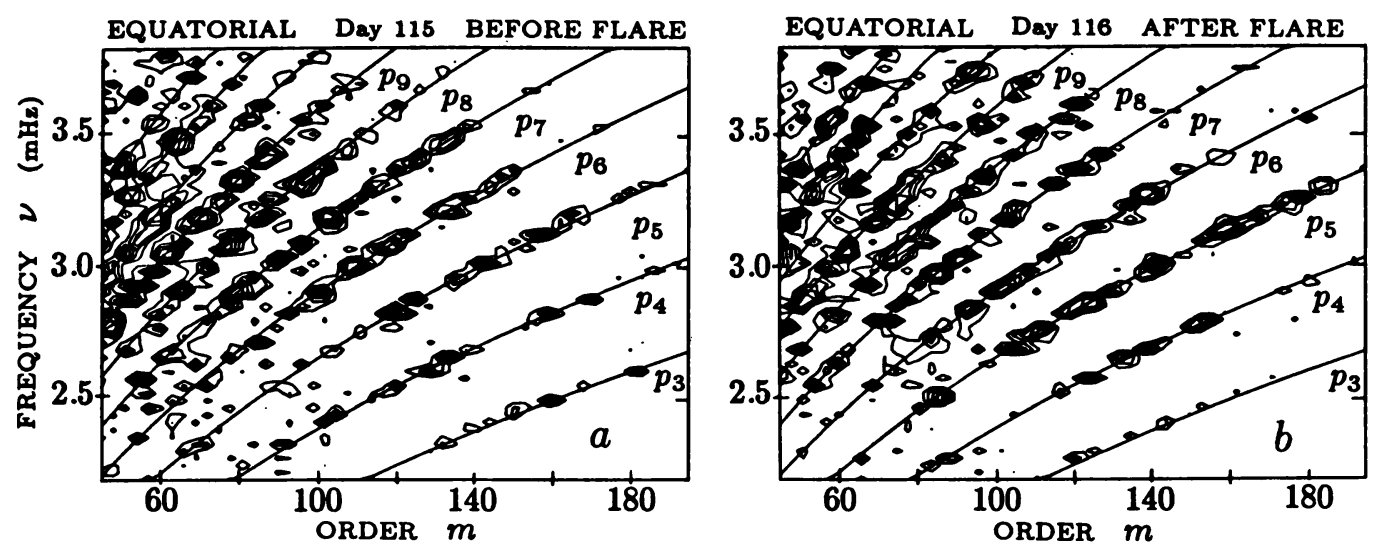

Figure 1. Power diagrams for equatorial sectoral modes for (a) Day 115 (before flare) and (b) Day 116 (after flare). Superposed are loci of frequencies from Harvey \& Duvall (1984) corrected for solar rotation. The $p_{5}$ ridge on Day 116 shows enhanced power.

\section{OBSERVATIONS AND DATA ANALYSIS}

Observations of intermediate-degree solar oscillations proceeded throughout Day 115, which ended with the flare, and the following Day 116. The UBF was used as an imaging Doppler analyzer, providing simultaneous images of the full Sun on two sides of the Fe I $\lambda 5576$ spectral line. These intensity images were recorded on $35 \mathrm{~mm}$ film at $60 \mathrm{~s}$ intervals in time and later digitized with 8 "spatial resolution. Doppler velocities were determined from ratios of the difference of a pair of intensity images to their sum. These velocities were calibrated by requiring that the solar sidereal equatorial rotation rate be the same within each image at about the Doppler rate from Mt. Wilson of $454 \mathrm{nHz}$ (Howard 1984). The velocities were finally interpolated onto a longitude-latitude grid (of $256 \times 256$ points) covering $120^{\circ}$ in each variable.

Two different types of modes were studied: sectoral modes travelling along the equator and similar modes propagating along a great circle aligned with the poles, much as considered in Hill et al. (1986). To look at the "equatorial" sectoral modes the data was first Fourier transformed in the east-west direction and then projected upon associated Legendre polynomials in the north-south direction. To isolate the "polar" sectoral modes a similar procedure was carried out but with the Fourier transform in the north-south direction and the Legendre projection in the east-west direction. To keep the analysis of the two days as identical as possible, the data sets were made the same length with equivalent time gaps before being Fourier transformed in time to form the $m-\nu$ power diagrams shown in Figure 1. The atmospheric seeing was comparable for both days, and given the 8 "spatial resolution, we do not expect the overall power levels to be dominated by seeing.

Power in the $m-\nu$ diagrams for the two days is compared for ridges with the same order $n$ (with say modes of $n=2$ denoted as $p_{2}$ ). Total power in a ridge is determined by drawing a narrow box around the ridge and then summing the power within that box. Identical boxes are used for both days. Judging by power levels in the ridges to that in the background, the signal-to-noise in this data is typically about 3.5:1. Therefore narrow boxes are needed to estimate the power in the modes contributing to a ridge. Table 1 presents ratios of the power from Day 116 to that in Day 115 for a variety of $n$ values, for both the positive and negative frequencies that correspond to differing directions of 
EQUATORIAL MODES

\begin{tabular}{|c|c|c|c|}
\hline $\begin{array}{l}\text { Radial } \\
\text { Order } \\
n\end{array}$ & $\begin{array}{c}\text { Day } \\
\text { 116/115 } \\
\text { Pos Freq }\end{array}$ & $\begin{array}{c}\text { Day } \\
\text { 116/115 } \\
\text { Neg Freq }\end{array}$ & $\begin{array}{c}\text { Day } \\
116 / 115 \\
\text { Pos + Neg Freq }\end{array}$ \\
\hline $\begin{array}{l}4 \\
5 \\
6 \\
7 \\
8 \\
9\end{array}$ & $\begin{array}{r}.93 \\
1.85 \\
1.10 \\
1.20 \\
.82 \\
.91\end{array}$ & $\begin{array}{r}.74 \\
.77 \\
.95 \\
1.21 \\
1.11 \\
.87\end{array}$ & $\begin{array}{r}.85 \\
1.35 \\
1.03 \\
1.20 \\
.90 \\
.90\end{array}$ \\
\hline Mean & $\overline{1.14}$ & $\overline{.94}$ & $\overline{1.04}$ \\
\hline
\end{tabular}

POLAR MODES

\begin{tabular}{|c|c|c|}
\hline $\begin{array}{l}\text { Day } \\
\text { 116/115 } \\
\text { Pos Freq }\end{array}$ & $\begin{array}{c}\text { Day } \\
116 / 115 \\
\text { Neg Freq }\end{array}$ & $\begin{array}{c}\text { Day } \\
116 / 115 \\
\text { Pos + Neg Freq }\end{array}$ \\
\hline 1.21 & 1.09 & 1.15 \\
\hline 1.16 & .81 & .98 \\
\hline .97 & 1.01 & .99 \\
\hline .90 & .78 & .85 \\
\hline .96 & 1.36 & 1.10 \\
\hline 1.20 & 1.04 & 1.13 \\
\hline$\overline{1.07}$ & $\overline{1.02}$ & $\overline{1.03}$ \\
\hline
\end{tabular}

Table 1. Power in ridges for Day 116 (after flare) divided by that on Day 115 (prior to flare) for both positive and negative frequencies, considering both equatorial and polar sectoral modes. The mean of these ratios over all $n$ values is also shown.

propagation for the modes. Those ratios where power for both senses of frequency have been summed are also reported.

\section{CONCLUSIONS}

The ratios of power levels in the various ridges as summarized in Table 1 suggest to us that typically $15 \%$ to $20 \%$ changes can occur from one day to another as measured within a given ridge. If the power in all the ridges is considered, there is an associated change of about $3 \%$ in the overall power ratio. We believe that such changes are consistent with what can result from beating effects between the many unresolved modes (of order 30) within each of the $m-\nu$ resolution bins along the ridges in the power spectra. We have examined some of our earlier oscillation data (cf. Hill, Gough, Toomre \& Haber, these proceedings) obtained with the diode-array instrument from one day to another for days with comparable atmospheric seeing; the ratios of power levels are consistent with the average trends seen in Table 1. This would lead us to conclude that there is no significant overall change in power levels of intermediate-degree oscillations on Day 116 following the flare from that of the quiet Sun on Day 115. However, there is one noteworthy exception. The $p_{5}$ ridge of positive frequencies for equatorial sectoral modes (propagating in the retrograde direction) is $85 \%$ higher in power on Day 116, showing an increase of about 3.5 standard deviations. We believe this substantial increase in power may be significant but we are hard put to explain why only the $p_{5}$ ridge should be so favored as a consequence of the flare.

The possible excitation of acoustic modes within the Sun by a major flare is a difficult subject to assess, for we have only fragmentary information about these nearly impulsive events in the atmosphere, and almost no data on how the disturbances may extend to deeper depths. The input of energy into the acoustic modes may proceed directly from compressional pulses generated by the flare. However, mechanical energy can also move downward as magnetohydrodynamic waves along the magnetic field bundles and contribute to the acoustic field. Yet that process is even more uncertain since the types of magnetic structures well below the surface are still a matter of some speculation. Such a variety of factors make it presently impossible to predict in any detail what a flare might do to excite, or to disrupt, certain global modes of oscillation. Certainly the energy released by the flare, compared to the energy inferred to be present in intermediate-degree modes, suggests that some effect is possible (Libbrecht et al. 1986). Further, given the horizontal scale of the flare region and the impulsive nature of repeated events within the flare, it is likely that a broad band of modes may be influenced. The unknown variation with depth of the flaring 
volume may lead to some values being favored over others, but here too the response is likely to be broad. Thus it is presently unclear why the $p_{5}$ modes alone appear to show what we deem to be a significant increase in power levels after the flare.

The differences in power for $p_{5}$ between positive and negative frequencies after the flare can perhaps be explained. Waves excited by the flare and travelling outward would appear in the observing window at different times due to the placement of the flare near the limb. When observations began on Day 116, those modes travelling prograde would be largely on the other side of the Sun while those moving retrograde would be coming onto the observable side. The waves are of course dispersive and their horizontal propagation speed depends on both $n$ and $\ell$, and thus what happens to wave packets is intricate, especially when considering how they project onto global modes of resonance. Yet we may expect there to be noticeable differences in power levels between retrograde and prograde modes for observations initiated about 12 hours after the flare on Day 116.

The oscillation data considered here were obtained with a preliminary setup of the $\mathrm{UBF}$, and required the use of film as the recording medium. The microdensitometry of such data has been tedious and time consuming, and we therefore have not finished reducing data from a few other days of observations in the same observing run. Such analysis should help place better bounds on the effects of mode beating that can lead to apparent changes in power along a ridge from one day to the next. Nevertheless, we find the substantial increase in power for the $p_{5}$ ridge after the flare to be rather enticing. That we were observing on the day of the flare was fortuitous and not easy to repeat. Thus we felt that the data, despite its difficulties, must be scrutinized. Clearly what is needed in the longer term is a data base such as the one to be produced by GONG where one should have a chance to study the effects of at least several flares with continuous coverage.

Acknowledgements - We thank Larry November for designing and implementing the new configuration of the UBF instrument, Don Neidig, Helen Coffey, and Pat McIntosh for helpful discussions about the observed properties of the flare, and Gary Wells for providing us with flare photographs. The Joint Institute for Laboratory Astrophysics is operated jointly by the National Bureau of Standards and the University of Colorado. The work at Colorado was supported in part by the National Aeronautics and Space Administration through grants NSG-7511, NAGW-91 and NAG-5-513, and by the Air Force Geophysics Laboratory through contract F19628-82-K-0008. The National Optical Astronomy Observatories are operated by the Association of Universities for Research in Astronomy, Inc. under contract with the National Science Foundation.

\section{REFERENCES}

Ando, H., and Osaki, Y. 1975, Pub. Astron. Soc. Japan 27, 581.

Goldreich, P., and Keeley, D.A. 1977a, Astrophys. J. 211, 934.

Goldreich, P., and Keeley, D.A. 1977b, Astrophys. J. 212, 243.

Harvey, J.W., and Duvall, T.L., Jr. 1984, in Theoretical Problems in Stellar Stability and Oscillations (eds. M. Gabriel and A. Noels; Observ. Liège), p. 209.

Hiei, E., Zirin, H., and Jingxiu, W. 1986, in The Lower Atmosphere of Solar Flares (ed. D.F. Neidig; National Solar Observatory, Sunspot), p. 129.

Hill, F., Haber, D.A., Toomre, J., and November, L.J. 1986, in Seismology of the Sun and the Distant Stars (ed. D.O. Gough; Reidel, Dordrecht), p.85.

Howard, R. 1984, Ann. Rev. Astron. Astrophys. 22, 131.

Libbrecht, K.G., Popp, B.D., Kaufman, J.M., and Penn, M.J. 1986, Nature 323, 235.

Svestka, Z. 1976, Solar Flares, (Reidel, Dordrecht).

Wolff, C.L. 1972, Astrophys. J. 176, 833. 